\title{
Relation eau de ruissellement et eau des sources et forages artésiens dans la région Zagnanado-Zogbodomey au Bénin : Approche couplée (pollution microbiologique - télédétection)
}

\author{
Gilbert Tite LALÈYÊ ${ }^{1 *}$, Expédit W. VISSIN ${ }^{2}$, Christophe Sègbédji HOUSSOU ${ }^{2}$ \\ et Patrick EDORH ${ }^{3}$ \\ ${ }^{1}$ École Doctorale Pluridisciplinaire "Espaces, Cultures et Développement", Faculté des Lettres, Arts et \\ Sciences Humaines, Université d'Abomey-Calavi (UAC), Cotonou, Benin. \\ ${ }^{2}$ Laboratoire Pierre Pagney - Climat-Eau-Écosystèmes-Développement Durable (LACEEDE) Faculté des \\ Lettres, Arts et Sciences Humaines, Université d'Abomey-Calavi (UAC), Cotonou, Benin. \\ ${ }^{3}$ Centre Interfacultaire de Formation et de Recherche en Environnement pour un Développement Durable \\ (CIFRED), Laboratoire de Biochimie et de Biologie cellulaire, Université d'Abomey-Calavi, Cotonou, Benin. \\ *Auteur correspondant : E-mail : Layetite2008@gmail.com; 04 BP 848 Cotonou, Benin ; Tel.: 22997530004
}

\section{RESUME}

La région Zagnanado-Zogbodomey constituée de grandes dépressions et de vallée a été retenue pour mieux comprendre l'influence des eaux de ruissellement sur la qualité des eaux souterraines des aquifères continus du Bassin sédimentaire côtier. A partir d'analyses microbiologiques portant sur des échantillons d'eau prélevés à la fois au niveau des sources et forages artésiens, la signature microbienne de chaque système hydrique a été établie. Les sources et forages artésiens sont plus affectés pendant la saison pluvieuse que la saison sèche, par la présence de germes bactériologiques. La présence de coliformes totaux et de coliformes fécaux dans l'eau des échantillons révèle que la contamination provient de la surface. Pour caractériser le seuil topographique qui représente une zone de recharge probable pour les eaux souterraines de la région, une étude des fractures à partir des linéaments observables sur un Modèle Numérique d'Altitude (MNA) détaillé de la région a été effectuée. En appui à la bactériologie, le traitement d'images satellitaires a permis de ressortir le réseau des fractures de notre zone d'étude située sur les formations du Bassin sédimentaire côtier. Les fractures étant en contact avec le réseau hydrographique, celles-ci pourraient jouer un rôle de drain pour les aquifères de la zone d'étude.

() 2014 International Formulae Group. All rights reserved.

Mots clés: Linéaments, infiltration, eau de ruissellement, eau souterraine.

\section{INTRODUCTION}

La plupart des nappes aquifères souterraines sont réalimentées par les eaux de ruissellement. Toutefois, dans le cas d'une source souterraine salubre, les eaux de ruissellement s'infiltrent à travers les terres de couverture (généralement formées d'un mélange de sols, de sable, de limon ou d'argile), puis dans le substratum rocheux. Cette filtration naturelle peut prendre des années. Puisque des bactéries telles que $E$. coli survivent pendant des semaines ou des mois dans l'eau et six mois ou plus dans le sol, elles devraient, en principe, disparaître de l'eau qui 
s'écoule et mourir durant le processus de filtration naturelle. D'après des études sur la durée de vie de E. Coli dans divers types de sols, les résultats montrent que de manière générale, le temps de survie dans le compartiment sol est très variable : il se compte parfois en semaine, mais aussi en mois, et même quelquefois en année, par exemple dans le cas d'un sol gelé (Blood et Henderson, 1968 in Majdoub et al., 2003 ; Givord et Dorioz, 2010). Si la source souterraine est salubre, la contamination bactérienne des eaux de ruissellement n'exerce aucune influence directe sur la nappe souterraine. Cependant, l'efficacité du processus de filtration naturelle peut dépendre de plusieurs facteurs, dont une liaison assez directe entre les eaux de ruissellement et la nappe aquifère. S'il existe un passage direct entre un puits ou un aquifère et les eaux de ruissellement, les bactéries vivantes peuvent pénétrer directement dans la source d'eau souterraine. On peut craindre qu'une activité se déroulant hors ou sur les terres de couverture des aquifères de la zone d'étude (les décharges sauvages, latrines inadéquates et centre d'enfouissement) contribue à la pollution des eaux de la nappe. L'étude de linéaments est réalisée depuis longtemps dans plusieurs domaines des sciences de la Terre que ce soit pour le pétrole, pour l'évolution géodynamique ou pour les applications hydrogéologiques (Anderson, 2009). Selon Sterckx (2013), les linéaments sont donc des structures linéaires identifiables sur des supports cartographiques et on pense qu'ils sont l'expression en surface de structures géologiques plus profondes. L'hypothèse utilisée dans cette étude est que les linéaments représentent le système de fracture que l'on retrouve dans la roche. Avec ces expressions linéaires, les informations que l'on peut obtenir sont nombreuses. Pour répondre à la problématique, des données cartographiques, des analyses bactériologiques des échantillons d'eaux des forages et sources artésiens pendant la saison sèche et la saison pluvieuse ont été réalisées. Les images Landsat TM de la scène Landsat OLI du 08/12/2013 ont été traitées pour étudier les linéaments. L'objectif du projet est d'étudier l'influence des eaux de ruissellement couplées à la fracturation de la zone Zagnanado-Zogbodomey à l'aide de la télédétection afin d'examiner les possibilités de transfert de polluants biologiques de la surface du sol vers les aquifères. Solomon et Ghebreab (2008) ont montré que les linéaments correspondant à des fractures de cisaillement influencent favorablement la productivité des puits à proximité.

\section{Contexte de l'étude}

Le cadre de l'étude composé des Communes de Zagnanado et de Zogbodomey au Bénin est compris entre $6^{\circ} 56^{\prime}$ et $7^{\circ} 30^{\prime} \mathrm{de}$ latitude Nord et $2^{\circ} 05^{\prime}$ et $2^{\circ} 25^{\prime}$ de longitude Est et couvre une superficie de $1575 \mathrm{~km}^{2}$ (IGN, 1992) (Figure 1). La présence des zones topographiques basses (vallées, dépressions) et la nature captive de l'aquifère du TuronienConiacien et de l'Eo-Paléocène favorisent la résurgence des eaux souterraines en plusieurs points dans les deux communes. Les sources artésiennes et l'aménagement des forages artésiens ainsi créées constituent une importante source d'approvisionnement pour les populations des deux communes.

Le relief de la commune de Zogbodomey est caractérisé par une plaine et un plateau qui couvre les $3 / 5$ de la superficie de la commune, avec un dénivelé d'environ $137 \mathrm{~m}$ entre le point le plus haut situé dans la portion nord de la commune et le point le plus bas sur la pointe Sud-est (PAGEFCOM, 2012). Ce plateau est situé au nord de la dépression de la Lama dont l'altitude moyenne atteint $50 \mathrm{~m}$ et la largeur variant de 5 $\mathrm{km}$ à l'Ouest à $25 \mathrm{~km}$ à l'Est. Ainsi, les pentes y sont généralement de moins de $5 \%$ (Figure 2). 
Le relief de la commune de Zagnanado est caractérisé par des plaines et des plateaux engendrant un dénivelé d'environ 270 m entre le point le plus haut situé dans la portion nord de la commune et le point le plus bas dans la pointe Sud. Les pentes y sont généralement de moins de $5 \%$, mais près de $4 \%$ du territoire est caractérisé par des versants avec une déclivité supérieure à 15\% (PAGEFCOM, 2012) (Figure 2).

Le régime pluviométrique dans les deux Communes est de subéquatorial. L'analyse de la pluviométrie et de la température à la station de Bohicon et de Zagnanado (1980-2012) révèle que les moyennes mensuelles sont respectivement de $1132,9 \mathrm{~mm}$ et de $1064 \mathrm{~mm}$ et $28{ }^{\circ} \mathrm{C}$.

\section{MATERIEL ET METHODES}

En ce qui concerne les données cartographiques, elles intéressent les cartes topographique et géologique à l'échelle 1/500 000 (Atalas, 1992). Du point de vue imagerie, les images Shuttle Radar Topography Mission (SRTM) fournies par le site américain ftp://easrp01u.ecs.nasa.gov/ ssrtm/version1/Africa ont été utilisées.

Les images du satellite Landsat TM de la scène OLI du 08/12/2013 sont téléchargées à partir du site " Earth Science Data Interface ». Les données des différentes cartes ont été traitées sous Arcview et l'image avec le logiciel Envi 4.7.

Pour le traitement du champ de fracturation (nombre et orientation des fractures) issues de l'image SRTM, nous avons utilisé le logiciel Linwin.

L'analyse bactériologique a porté sur la recherche de germes indicateurs de pollution biologiques tels que les germes banaux, les coliformes totaux, les coliformes thermotolérants (Escherichia coli), les streptocoques fécaux et les Clostridium sulfito-réducteurs. Les analyses des échantillons ont été réalisées au Laboratoire de Contrôle Qualité des Eaux et Aliments (LCQEA) de la Direction Nationale de Santé Publique (DNSP) au ministère de la Santé (Bénin).

\section{La cartographie des linéaments par télédétection}

Le terme linéament désigne toutes structures linéaires qui se marquent dans la topographie (Hobbs, 1904 in Hung et al., 2005). Celui-ci peut indiquer la variation de la résistance de roche (Scanvic, 1993 in Babaye, 2012) ou la présence d'une fracture.

La cartographie des linéaments peut se faire par analyse des cartes topographiques, des réseaux hydrographiques ou par interprétation des photographies aériennes (Yaméogo, 2008). Ces dernières années, les hydrogéologues utilisent de plus en plus les supports numériques tels le MNT (Durand, 2005) et les images satellitaires (Saley, 2005 ; Gronayes et al., 2003 ; Jourda, 2005) pour l'extraction des linéaments. Dans cette étude, nous utilisons les images satellitaires pour cartographier les linéaments dans la région de Zagnanado et de Zogbodomey. La méthodologie développée dans cette partie permettra d'accentuer et d'extraire les linéaments structuraux identiques à partir des images satellitaires.

\section{Filtre directionnel}

Pour accentuer les contrastes entre les structures de l'image, nous lui avons appliqué le filtre directionnel de convolution de matrice $3 \times 3$ selon les orientations N-S, NE-SW, EW et NW-SE. Ainsi, pour chaque direction utilisée, le filtre accentue la direction qui lui est perpendiculaire. La Figure $3 \mathrm{a}$ et $3 \mathrm{~b}$ montre l'exemple d'une image brute SRTM et son aspect après passage sous le filtre de convolution-directionnel. 


\section{Extraction des linéaments du secteur d'étude}

L'extraction des linéaments peut se faire automatiquement par utilisation d'un algorithme mathématique (Hung et al., 2005). A la fin des filtrages, les images résultantes sont importées sous le fichier type du logiciel PCI Géomatica. A l'aide de ce dernier, nous passons une à une les images sous l'outil regroupant les algorithmes des divers traitements. Ce traitement a permis d'extraire autant de linéaments que d'images filtrées selon les différentes directions. La Figure 4 montre un réseau de linéaments issu de l'extraction. Chaque couleur des lignes présentées sur cette figure correspond à une extraction selon une direction choisie. Pour cartographier ces linéaments, il a été procédé à une vectorisation de chaque extraction. Après, les vecteurs ont été exportés sous le fichier type d'Arc Gis.

\section{Contrôle et validation}

La phase de contrôle et de validation des structures linéaires extraites à partir du traitement des images est nécessaire pour préciser leur signification structurale (Razack, 1984 in Babaye, 2012). Une fois la carte de linéaments réalisée (Figure 6), il faut la valider et donc montrer que les linéaments majeurs repérés sur l'image radar sont en accord avec la fracturation (relevée à l'affleurement et sur carte géologique) des formations géologiques du site d'étude. Cette validation est basée sur la comparaison des directions des grandes familles de linéaments (issus du traitement de l'image radar) avec les directions de fracturation relevées sur carte géologique. L'obtention d'éléments de validation pour les linéaments est difficile. En effet, toutes les fractures ne sont pas nécessairement visibles à l'affleurement, ou sont masquées par un couvert végétal parfois dense en milieu tropical. Malgré ces différentes limitations, la validation des linéaments est réalisée sur la base de données de fracturations relevées sur carte géologique. La superposition de la carte des linéaments aux données géologiques montre qu'il a une corrélation entre ces deux types de données (Figure 6). Les linéaments majeurs correspondent aux failles d'envergure régionales. En plus de cette similitude, la carte des linéaments est plus dense que celle des failles. Cette carte a une importance appréciable en hydrogéologie. Elle permet de mettre en évidence les nœuds de fractures d'une part, et d'autre part les couloirs potentiels d'écoulement souterrain.

\section{Analyse bactériologique}

Les résultats des analyses bactériologiques, obtenus après deux campagnes, sont présentés dans les Tableaux (2 et 3). Ces analyses portent sur les échantillons d'eaux de 7 sources artésiennes à Zagnanado (où existent des sources artésiennes) et de 5 forages artésiens à Zogbodomey (où existent uniquement des forages), captant les aquifères des sables du Crétacé supérieur, répartis sur l'ensemble de la zone d'étude. Le choix des sources et forages artésiens de prélèvement des eaux échantillonnées a reposé sur plusieurs critères à savoir :

proximité du site par rapport aux habitations ;

- densité des populations qui s'y approvisionnent ;

- forme d'aménagement des sources artésiennes.

Singulièrement pour les forages, le choix a porté sur un forage par arrondissement.

Les analyses sont effectuées au Laboratoire suivant le protocole décrit dans le Tableau 1. 


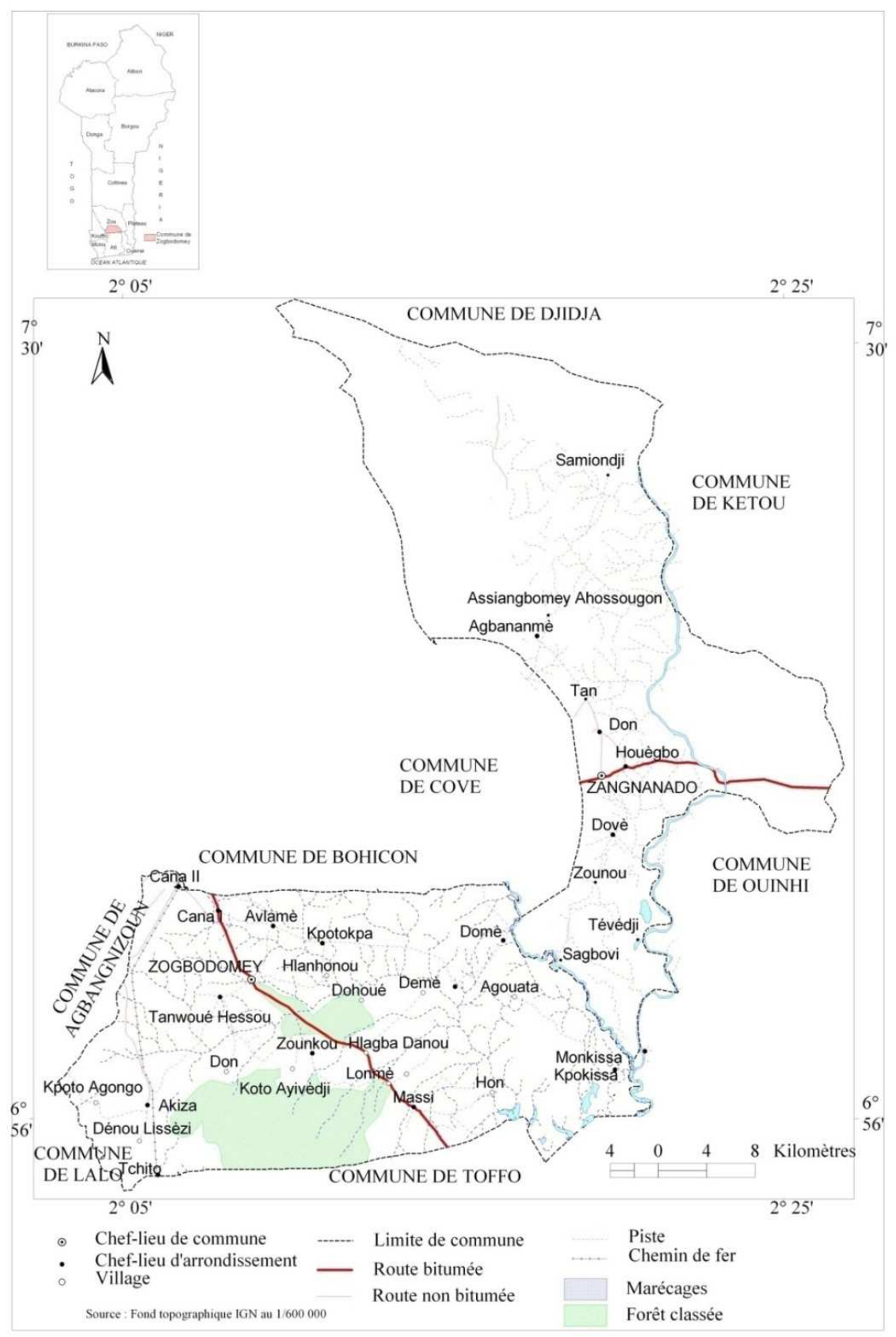

Figure 1 : Situation géographique et cadre physique.

\section{RESULTATS}

L'analyse des résultats du Tableau 2 montre que la quasi-totalité des eaux des forages artésiens sont polluées du point de vue microbiologique. Cependant, les Coliformes fécaux des streptocoques fécaux, et les E. coli qui sont considérés comme plus appropriés de révéler des contaminations fécales sont absents dans les eaux des forages artésiens en saison sèche. 
L'analyse des résultats du Tableau 3 montre que la totalité des eaux des sources artésiennes sont polluées du point de vue microbiologique. D'une manière générale, les valeurs des coliformes totaux, des streptocoques fécaux, et des coliformes fécaux sont largement au-dessus de la norme
OMS (2006) dans tous les échantillons d'eau des sources artésiennes, en saison pluvieuse qu'en saison sèche. Ceci dénote d'une pollution d'origine fécale. Cette pollution est d'autant plus élevée que ce sont des activités de baignade, de lessive et de vaisselle qui sont menées dans l'eau de ces sources.

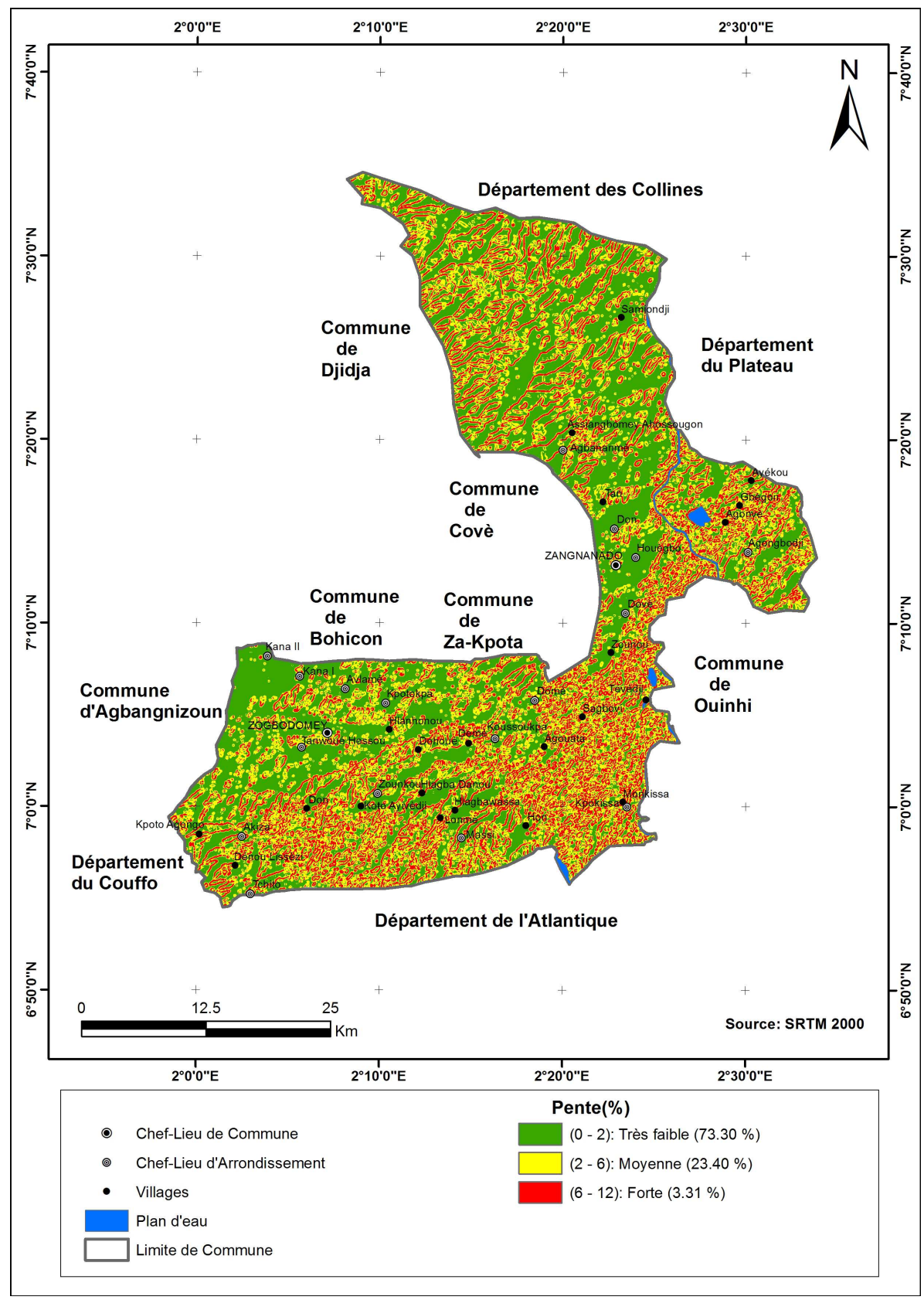

Figure 2 : Pente dans les communes de Zagnanado et de Zogbodomey. 


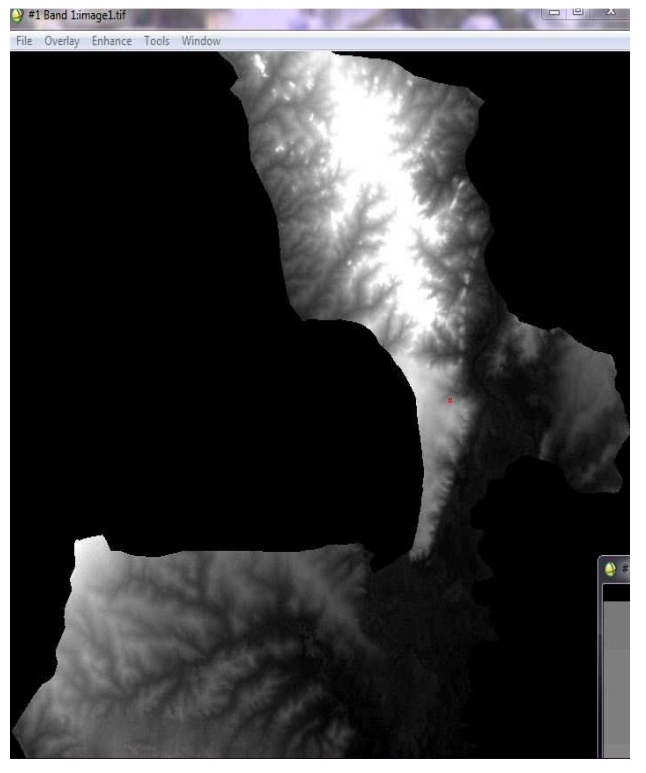

(a)

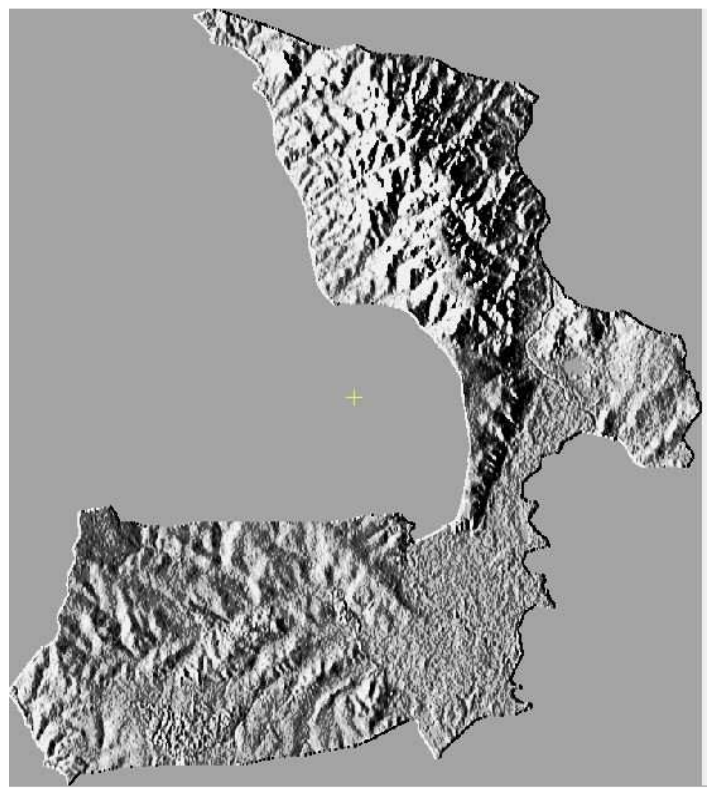

(b)

Figure 3 : Image brute(a) et filtrée(b).

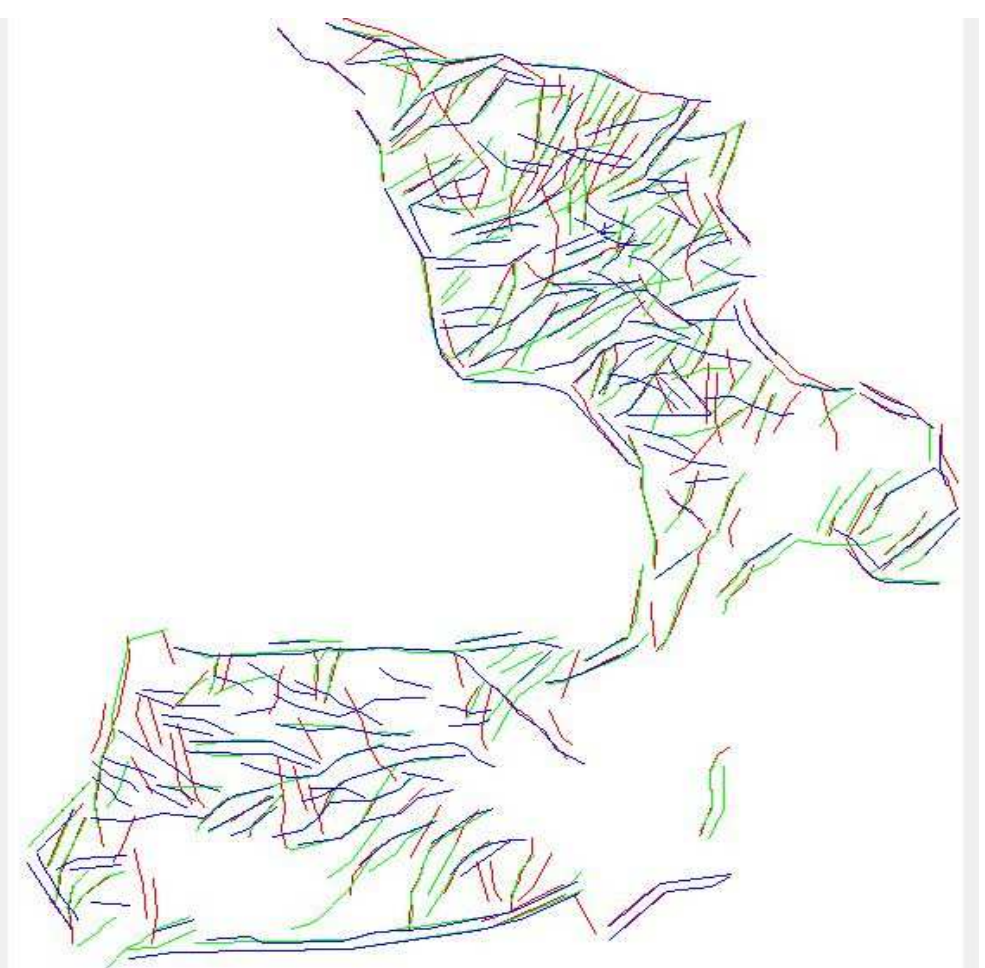

Figure 4 : Carte de réseau de linéaments issu de l'extraction. 

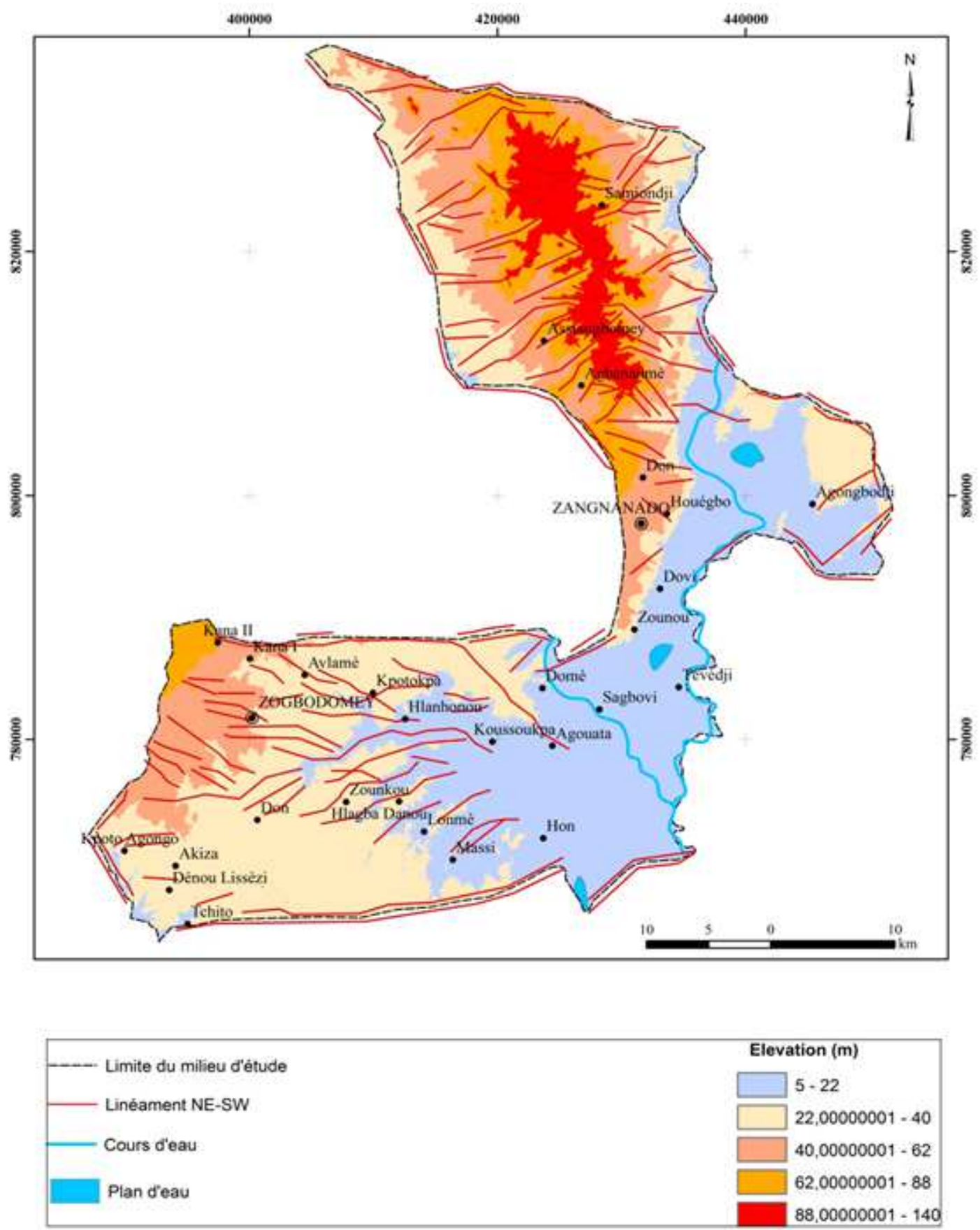

Source : Image SRTM, 2013.

Figure 5: Linéaments / fracturation à Zogbodomey et Zagnanado au Benin. 


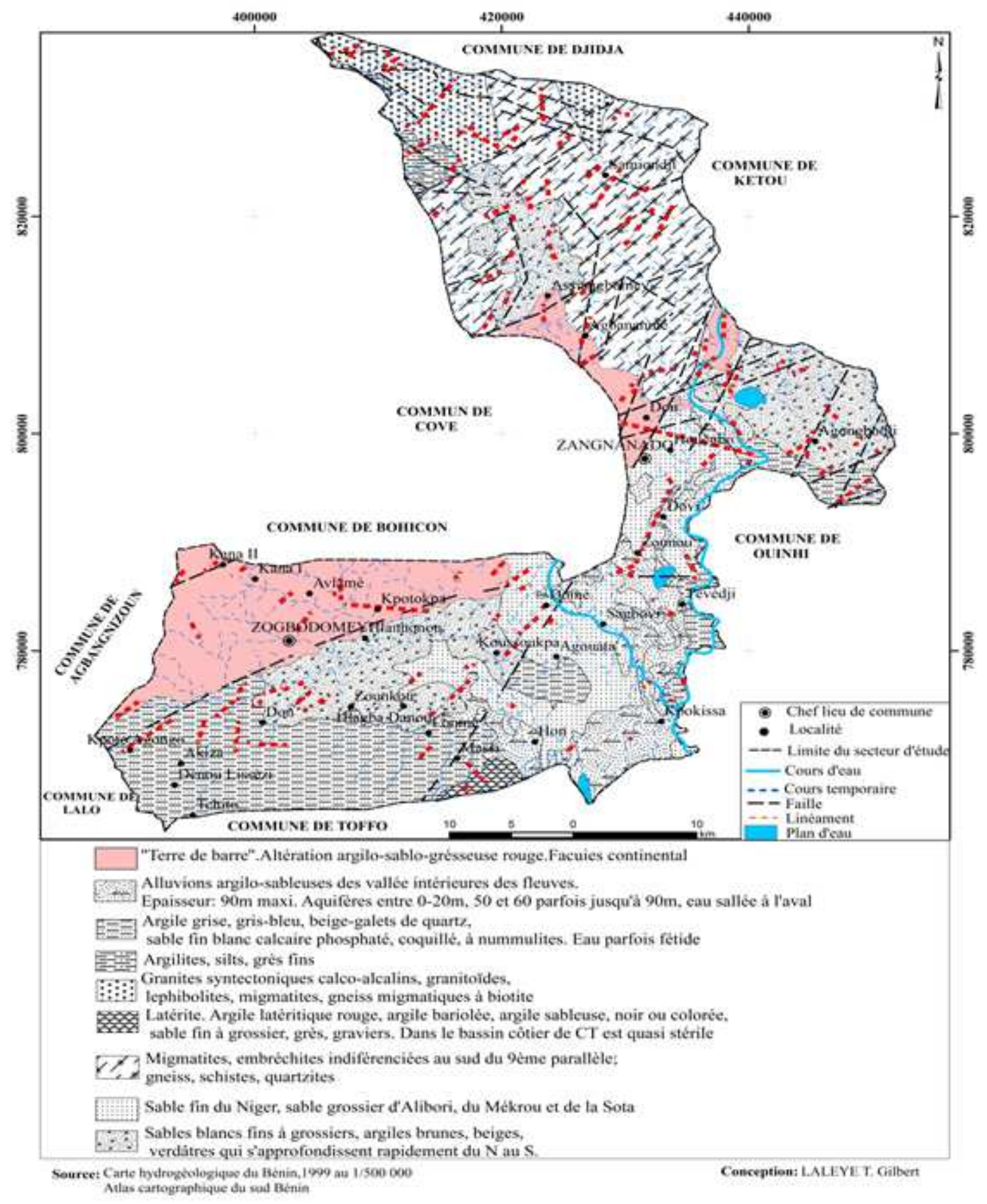

Figure 6: Relation linéaments et factures issues des cartes d'hydrogéologies. 
Tableau 1 : Protocole d'analyse bactériologique des eaux de forages et de sources artésiens Prélevées dans les deux communes.

\begin{tabular}{|c|c|c|c|}
\hline Milieu & Mode d'utilisation & $\begin{array}{c}\text { Température } \\
\text { d'incubation et temps }\end{array}$ & $\begin{array}{c}\text { Colonies } \\
\text { identifiées }\end{array}$ \\
\hline $\begin{array}{l}\text { Gélose nutritive } \\
\text { pour le } \\
\text { dénombrement des } \\
\text { germes banals ou } \\
\text { de la flore totale }\end{array}$ & $\begin{array}{l}\text { - Mélange soigneux et chauffage doux avec } \\
\text { agitation continue sur agitateur magnétique } \\
\text { - Ebullition pendant une à deux minutes } \\
\text { - Répartition et stérilisation à l'autoclave à } 121^{\circ} \mathrm{C} \text { pendant } \\
15 \text { minutes. } \\
\text { La gélose est coulée par incorporation. }\end{array}$ & $\begin{array}{l}37^{\circ} \mathrm{C} \text { pendant } 24 \\
\text { à } 48 \text { heures }\end{array}$ & $\begin{array}{l}\text { Toutes les } \\
\text { colonies sans } \\
\text { distinction. }\end{array}$ \\
\hline $\begin{array}{l}\text { Gélose nutritive Rapid'E.coli pour } \\
\text { le dénombrement des Coliformes }\end{array}$ & $\begin{array}{c}\text { - Dissoudre la poudre du REC dans l'eau distillée ; } \\
\text { - Porter à ébullition lentement sous agitation magnétique ; } \\
\text { Autoclaver ou pas à } 121^{\circ} \mathrm{c} \text { pendant } 15 \mathrm{mn} \text {; } \\
\text {-Appliquer la technique de filtration sur membrane } \\
\text { cellulosique de porosité } 0,45 \mu \mathrm{m} \text {. }\end{array}$ & $\begin{array}{l}37^{\circ} \mathrm{C} \text { pendant } 24 \\
\text { à } 48 \text { heures pour } \\
\text { les Coliformes } \\
\text { totaux et } 44^{\circ} \mathrm{C} \\
\text { pour les thermo } \\
\text { tolérants }\end{array}$ & $\begin{array}{c}\text { Colonies bleues sont des } \\
\text { Coliformes et les violettes sont les } \\
\text { E. coli }\end{array}$ \\
\hline $\begin{array}{l}\text { SLANETZ et } \\
\text { BARTLEY pour } \\
\text { les Streptocoques }\end{array}$ & $\begin{array}{l}\text { - Dissoudre la poudre du SLANETZ dans l'eau distillée } \\
\text { et chauffer pendant } 20 \text { minutes à l'autoclave (sans } \\
\text { pression); } \\
\text { - Refroidir entre } 45 \text { et } 50^{\circ} \mathrm{C} \text {. } \\
\text { - Répartir dans des boîtes de pétri pour solidification. } \\
\text { - Appliquer la technique de filtration sur membrane } \\
\text { cellulosique de porosité } 0,45 \mu \mathrm{m} \text {. }\end{array}$ & $\begin{array}{c}36 \pm 2{ }^{\circ} \mathrm{C} \text { pendant } \\
44 \pm 4 \text { heures }\end{array}$ & $\begin{array}{c}\text { Colonies } \\
\text { convexes rouges, } \\
\text { roses ou marron, } \\
\text { avec ou sans } \\
\text { auréole blanche. } \\
\text { Si le résultat est positif, il faut faire } \\
\text { la conformation sur le milieu BEA }\end{array}$ \\
\hline $\begin{array}{l}\text { TSN pour les sulfitoréducteurs } \\
\text { (Clostridium perfringens) }\end{array}$ & $\begin{array}{l}\text { - Dissoudre la poudre du TSN dans l'eau distillée ; } \\
\text { Porter à ébullition lentement sous agitation magnétique ; } \\
\text { - Stériliser à l'autoclave à } 121^{\circ} \mathrm{C} \text { pendant } 15 \\
\text { Minutes ; } \\
\text {-Faire un ensemencement profond en tubes ou dans des } \\
\text { boîtes de pétri. }\end{array}$ & $\begin{array}{c}37^{\circ} \mathrm{C} \text { pendant } 24 \mathrm{~h} \text { en } \\
\text { anaérobiose }\end{array}$ & Colonies noires entourées de halo. \\
\hline
\end{tabular}


Tableau 2 : caractéristiques bactériologiques des eaux des forages artésiens en saisons pluvieuse et sèche.

\begin{tabular}{|c|c|c|c|c|c|c|c|}
\hline Arrondissement & VMA & Période & Kpokissa & Avlamey & Koussoukpa & Zoukou & Massi \\
\hline Lieu de prélèvement & & & Kpokissa Centre & Avlamey & Samionta & Hlanhonou & Hon \\
\hline Echantillon & & & & Gbata & EPP Samionta & & Hon \\
\hline \multirow[t]{2}{*}{ Date } & & Saison pluvieuse & $31 / 08 / 2013$ & $31 / 08 / 2013$ & $31 / 08 / 2013$ & $31 / 08 / 2013$ & $31 / 08 / 2013$ \\
\hline & & Saison sèche & $08 / 04 / 2013$ & $08 / 04 / 2013$ & $08 / 04 / 2013$ & $08 / 04 / 2013$ & $08 / 04 / 2013$ \\
\hline \multirow{2}{*}{ Dénombrement total des germes banals par mL } & 50 & Saison pluvieuse & 152 & 230 & 1400 & 500 & 216 \\
\hline & & Saison sèche & 00 & 01 & 00 & 00 & 00 \\
\hline \multirow{2}{*}{ Colimétrie par $100 \mathrm{~mL}$ : Coliformes totaux } & 0 & Saison pluvieuse & 01 & 57 & 119 & 76 & 125 \\
\hline & & Saison sèche & 00 & 02 & 03 & 00 & 00 \\
\hline \multirow[t]{2}{*}{ Colimétrie par $100 \mathrm{~mL}$ : Coliformes fécaux } & 0 & Saison pluvieuse & 00 & 03 & 27 & 09 & 71 \\
\hline & & Saison sèche & 00 & 00 & 00 & 00 & 00 \\
\hline \multirow{2}{*}{ Colimétrie par $100 \mathrm{~mL}$ : Escherichia coli } & 0 & Saison pluvieuse & 00 & 00 & 00 & 00 & 26 \\
\hline & & Saison sèche & 00 & 00 & 00 & 00 & 00 \\
\hline \multirow{2}{*}{ Streptocoques fécaux par $100 \mathrm{~mL}$} & 0 & Saison pluvieuse & 12 & 96 & 34 & 00 & 33 \\
\hline & & Saison sèche & 00 & 00 & 00 & 00 & 00 \\
\hline \multirow{2}{*}{ Clostridium perfringens par $20 \mathrm{~mL}$} & 02 & Saison pluvieuse & 00 & 00 & 00 & 00 & 00 \\
\hline & & Saison sèche & 00 & 00 & 00 & 00 & 00 \\
\hline
\end{tabular}


Tableau 3 : Caractéristiques bactériologiques des eaux des sources artésiennes en saisons pluvieuse et sèche.

\begin{tabular}{|c|c|c|c|c|c|c|c|c|c|}
\hline Arrondissement & VMA & Période & Zagnanado & Agonlin & Banamè & Dovi & Don-Tan & & \\
\hline Lieu de prélèvement & & & Doga & Dohounmè & Houégbo & Ahandatin & Kahodji & Dovi-dovè & \\
\hline Echantillon & & & Massavi & Wakpa & N'gbèdè & Ahandatin & & Dowè & Tovè \\
\hline \multirow{2}{*}{ Date } & & Saison pluvieuse & $17 / 06 / 2013$ & $17 / 06 / 2013$ & $17 / 06 / 2013$ & $17 / 06 / 2013$ & $17 / 06 / 2013$ & $17 / 06 / 2013$ & $17 / 06 / 2013$ \\
\hline & & Saison sèche & $08 / 04 / 2012$ & 08/04/2012 & $08 / 04 / 2012$ & 08/04/2012 & 08/04/2012 & 08/04/2012 & 08/04/2012 \\
\hline \multirow{2}{*}{$\begin{array}{l}\text { Dénombrement total des } \\
\text { germes banals par } \mathrm{mL}\end{array}$} & 50 & Saison pluvieuse & 214 & 900 & 24 & 7200 & 3040 & 8300 & 1250 \\
\hline & & Saison sèche & 21 & 4510 & 92 & 532 & 260 & 2125 & 2300 \\
\hline \multirow{2}{*}{$\begin{array}{l}\text { Colimétrie par } 100 \mathrm{~mL}: \\
\text { Coliformes totaux }\end{array}$} & 0 & Saison pluvieuse & 1220 & 1960 & 140 & 24000 & 4800 & 6400 & 5600 \\
\hline & & Saison sèche & 60 & 5640 & 440 & 6800 & 2960 & 2860 & 3040 \\
\hline \multirow{2}{*}{$\begin{array}{l}\text { Colimétrie par } 100 \mathrm{~mL}: \\
\text { Coliformes fécaux }\end{array}$} & 0 & Saison pluvieuse & 420 & 198 & 36 & 380 & 900 & 1000 & 700 \\
\hline & & Saison sèche & 00 & 980 & 00 & 220 & 340 & 740 & 480 \\
\hline \multirow{2}{*}{$\begin{array}{l}\text { Colimétrie par } 100 \mathrm{~mL} \text { : } \\
\text { Escherichia coli }\end{array}$} & 0 & Saison pluvieuse & 16 & 64 & 00 & 220 & 300 & 200 & 260 \\
\hline & & Saison sèche & 00 & 40 & 00 & 100 & 110 & 180 & 400 \\
\hline \multirow{2}{*}{$\begin{array}{l}\text { Streptocoques fécaux par } 100 \\
\mathrm{~mL}\end{array}$} & 0 & Saison pluvieuse & 05 & 70 & 06 & 52 & 236 & 98 & 268 \\
\hline & & Saison sèche & 20 & 140 & 00 & 40 & 70 & 40 & 220 \\
\hline \multirow{2}{*}{$\begin{array}{l}\text { Clostridium perfringens par } \\
20 \mathrm{~mL}\end{array}$} & 02 & Saison pluvieuse & 00 & 04 & 00 & 16 & 72 & 00 & 16 \\
\hline & & Saison sèche & 00 & 20 & 00 & 16 & 20 & 08 & 60 \\
\hline
\end{tabular}




\section{DISCUSSION}

Dans la région de Zagnanado et de Zogbodomey, le résultat des analyses microbiologiques indique qu'au niveau des sources artésiennes les germes sont présents et nombreux. Les germes banaux et les coliformes totaux sont innombrables dans la totalité des eaux. Le nombre d'Escherichia coli excède aussi les normes de l'OMS et sont de 16 à 260 UFC/100 ml et 00 à 400 UFC/100 ml, respectivement en saison pluvieuse et en saison sèche. Les streptocoques fécaux sont de 5 à $268 \mathrm{UFC} / 100$ $\mathrm{ml}$ en saison pluvieuse et 20 à $220 \mathrm{UFC} / 100$ $\mathrm{ml}$ en saison sèche. . Les Clostridium perfringens sont aussi présents et varient de 0 à160 UFC/100 ml et 0 à 60 UFC/100 ml respectivement en saison pluvieuse et en saison sèche. Les résultats similaires ont été trouvés par Totin (2010) dans le bassin sédimentaire côtier du Bénin. La présence de coliformes totaux et de coliformes fécaux dans l'eau des échantillons des sources artésiennes révèle que la contamination provienne de la surface.

En ce qui concerne les eaux des forages, ils sont aussi affectés par la présence de germes bactériologiques tels que les Coliformes totaux, les Coliformes fécaux, et les Streptocoques fécaux dans la totalité des eaux de forages artésiens analysées, pendant la saison pluvieuse. Les streptocoques fécaux sont détectés et leur nombre varie dans les eaux des forages $(0$ à $96 \mathrm{UFC} / 100 \mathrm{ml})$. Le nombre des Coliformes fécaux excède aussi les normes de l'OMS et sont de 0 à 71 UFC/100 ml. Le forage de Hon est le plus affecté des forages par la présence élevée de germes bactériologiques. On enregistre au niveau de ce forage la présence des d'Escherichia coli dont le nombre s'élève à $26 \mathrm{UFC} / 100 \mathrm{ml}$. La présence de coliformes totaux, de coliformes fécaux et de streptocoques fécaux dans l'eau des échantillons des forages artésiens révèle que la contamination provienne de la surface. La présence de ces germes uniquement en saison des pluies au niveau des forages et leur importance au cours de la même saison au niveau des sources atteste l'influence des eaux de ruissellement sur la qualité des eaux. Les bactéries fécales, y compris E. coli, sont présentes dans l'eau brute. Étant donné la durée de vie assez brève de ces organismes, la présence de bactéries fécales dans une nappe souterraine signale l'existence, en surface, d'un point de contamination fécale à une distance pouvant être parcourue rapidement, ainsi qu'une filtration naturelle inadéquate par les matières souterraines entourant la grille d'entrée du point d'eau. Toutefois, dans le cas d'une source souterraine salubre, les eaux de ruissellement s'infiltrent à travers les terres de couverture (généralement formées d'un mélange de sols, de sable, de limon ou d'argile), puis dans le substratum rocheux. Cette filtration naturelle peut prendre des années.

Cependant, l'efficacité du processus de filtration naturelle peut dépendre de plusieurs facteurs, dont une liaison assez directe entre les eaux de ruissellement et la nappe aquifère. S'il existe un passage direct entre un puits ou un aquifère et les eaux de ruissellement, les bactéries vivantes peuvent pénétrer directement dans la source d'eau souterraine.

Les résultats obtenus de l'étude des linéaments sur la zone d'étude tendent donc à démontrer qu'il y a une hétérogénéité dans la densité des linéaments dans les communes de Zagnanado et de Zogbodomey (Figure 5). La relation entre l'observation des linéaments et la pollution microbienne pendant la saison pluvieuse est sans ambiguité.

Les zones fracturées résultant principalement de phénomènes tectoniques. Pléthore d'études ont mis en évidence la bonne corrélation entre la productivité des puits et les discontinuités détectées par les linéaments (Fernandes and Rudolph, 2001; Kouamé et al. 2010; Naik et al,. 2001; Neves and Morales, 2007). Parmi ces études, un bon nombre se concentre sur la variation de la productivité des puits avec l'orientation des linéaments, l'idée étant d'établir un lien entre des événements tectoniques et la productivité (Fernandes and Rudolph, 2001; Neves et Morales, 2007). La position des linéaments étant ainsi perçue comme un des indicateurs de la ressource en eau, elle est classiquement intégrée dans le processus de création de cartes de productivité potentielle (Elewa and 
Qaddah, 2011; Lee et al., 2012; Masoud et Koike, 2005; Oh et al., 2011; Rao, 2006).

Selon Braathen et Gabrielsen (1998), in Babaye, 2012, un grand linéament peut améliorer la fracturation jusqu'à $300 \mathrm{~m}$ tandis que Clark (1985, in Babaye, 2012) a suggéré que la zone d'influence pourrait être moins de $150 \mathrm{~m}$ puis Fernandes et Rudolph (2001) réduisent celle-ci à $70 \mathrm{~m}$ dans leurs études. Les aquifères de la zone d'étude sont à moins de $150 \mathrm{~m}$ et peuvent être considérés comme ciblant un linéament. En utilisant l'hypothèse que les linéaments correspondent à des structures cassantes dans le socle rocheux, l'intersection apporte de l'information importante. Une plus forte intersection de linéaments et une plus forte densité indiquent donc des endroits où l'eau souterraine pourrait circuler davantage. La commune de Zagnanado est donc considérée comme une zone plus fracturée et donc plus perméable. La singularité des forages de Gbata et d'EPP Samionta où on enregistre la présence peu élevé de coliformes totaux pendant la saison sèche serait en réalité liée au fait à la dégradation de la dalle anti bourbier des ouvrages. Le phénomène entraîne une érosion en dessous de la dalle. La stabilité des tubages internes et de la protection de surface n'est plus assurée. Les activités autour du point d'eau réalisées par les hommes (baignade) et les femmes (lessive et parfois vaisselle) polluent l'eau avec l'effet de mousse.

Par ailleurs, les sources sont les exutoires naturels des nappes. Pendant longtemps, elles ont été captées sans être aménagées. L'eau est canalisée par gravité vers le lieu d'utilisation. La plupart de ces sources artésiennes très importantes dans la commune de Zagnanado ne sont pas aménagées. Elles se situent dans de grandes dépressions (4\% du territoire est caractérisé par des versants avec une déclivité supérieure à 15\%). Dans la nappe, l'eau circule jusqu'à des exutoires qui sont dans les points bas de la topographie. La pente topographique qui influence l'écoulement superficiel des eaux : ruissellement de surface et écoulement hypodermique, accélère le ruissellement sur les versants jusqu'à des exutoires qui sont dans les points bas de la topographie (sources, rivières) et détermine en partie le temps de réponse du cours d'eau aux impulsions pluviométriques.

Aussi, faut-il soulever le rôle que jouent les brèches qui se retrouvent sous les troncs d'arbres qui séparent la partie réservée aux activités domestiques et la partie où on prélève l'eau de consommation. Ces sources sont de véritables lieux d'activités domestiques de toutes sortes comme la lessive, la vaisselle et aussi de baignade. En effet, les substances toxiques naturelles et celles produites par les activités domestiques sur les sites polluent l'eau de ces sources. Les déchets issus de la lessive, de la baignade, de la vaisselle, et autres activités contribuent ainsi à l'augmentation de la concentration des matières en suspension dans l'eau donc à sa pollution.

La variation de charge polluante enregistrée en saison pluvieuse illustre l'influence du climat sur la qualité des eaux étudiées. La température et la pluie, en l'occurrence l'eau de ruissellement, interviennent directement dans le processus bactériologique, de développement des agents pathogènes qui, par infiltration à travers les linéaments (un passage direct entre un aquifère et les eaux de ruissellement) en saison pluvieuse, affectent les eaux des forages et sources artésiens.

Les organismes se trouvant dans l'eau des sources et forages artésiens peuvent provenir des couches de protection partielles contaminées par des déchets (excréments d'oiseaux et de mammifères en particulier) ou par des personnes ou des animaux infectés. Sur le plan topographique, les sources se retrouvent dans les vallées ou des dépressions. Dans ces conditions, l'eau de ruissellement est probablement à l'origine de la pollution de l'aquifère par le mécanisme de migration, à travers une zone fracturée et érodée et aussi vers l'exutoire des sources. L'infiltration ponctuelle à cause de brèches dans les terres 
de couverture protectrices, suivie d'une migration rapide vers le substratum rocheux semble être une explication plausible de la présence des germes fécaux dans les eaux des forages et sources artésiens.

La présence de Clostridium perfringens dans les eaux des sources artésiennes, hormis les sources Massavi et N'gbèdè, est une solution pour constater qu'elles ont bel et bien subit une contamination fécale. De même, la présence des Streptocoques fécaux au niveau des mêmes sources et des forages témoigne d'une contamination fécale ancienne.

Par ailleurs, la présence des Escherichia coli dans l'eau du forage de Hon et des sources prélevées, hormis les sources de Massavi et N'gbèdè, indique non seulement une contamination récente par les matières fécales, mais aussi la présence possible de bactéries, virus et protozoaires pathogènes.

La vulnérabilité des eaux des sources et forages artésiens à la pollution dépend d'une part des possibilités de transits de la pollution, de la surface du sol vers la nappe et d'autre part de la propagation des polluants provenant des activités humaines.

En plus de la bonne corrélation établie entre les linéaments et la productivité des puits, la nature même des linéaments - liés à la surface - les rend facilement accessibles et justifie la systématisation de leur exploration. L'approche classique d'exploration de la ressource en eau consiste donc à repérer ces accidents affleurant en surface, issus majoritairement de zones sub-verticales (Singhal et Gupta, 2010) ; l'emploi des méthodes de télédétection y est prépondérant (Sander, 2007; Singhal et Gupta, 2010). Ces linéaments jouent un rôle de drain (fonction conductrice). Elle est aussi très variable et il est très courant de rencontrer des puits « secs », car les fractures ne sont pas systématiquement ouvertes et connectées (Kuusela- Lahtinen et al., 2003; Neves et Morales, 2007; Walker et al., 2001) et sont limitées dans leur extension. En tant que structures que l'on peut qualifier de quasi bidimensionnelles, les fractures ne disposent pas d'un coefficient de stockage élevé (Le Borgne et al., 2006). Aux problèmes de connectivité et à leur caractère local, s'ajoute leur sub-verticalité qui limite a priori la communication avec des structures environnantes et la collecte de la recharge (Sander, 2007). Plus récemment, la zone altérée a été identifiée comme l'autre structure porteuse d'une ressource en eau. Sa formation est un phénomène complexe : le type d'altération résulte en un matériau contribuant à l'existence d'une ressource (augmentation de la porosité) et/ou l'entravant (formation d'argile) selon le climat et le type de roche (Singhal and Gupta, 2010). Comme dit précédemment, les zones fracturées peuvent constituer des zones d'altération préférentielle en ce qu'elles peuvent faciliter l'infiltration de l'eau en profondeur, même si la zone altérée reste assez peu profonde. Tout ceci résulte tant en des caractéristiques hydrodynamiques et structurales très variables dans l'espace, qu'en une ressource incertaine (Taylor et Howard, 2000). Aussi, la proximité avec la surface accroît leur vulnérabilité aux contaminations anthropiques (Dewandel et al., 2010). Pourtant, cette structure constitue parfois la seule ressource disponible (Negrel et al., 2011). Sa caractérisation et celle de la ressource associée passe par la régionalisation de ses propriétés - perméabilité et porosité (traitement statistique des données avec ou sans inversion, lien entre les mesures géophysiques et les paramètres hydrodynamiques) empruntée à la caractérisation des aquifères alluviaux et sédimentaires (Dewandel et al., 2012). L'organisation en strates (ou multicouches) de cette structure justifie ce rapprochement avec les milieux poreux.

\section{Conclusion}

La qualité bactériologique des eaux des forages et sources artésiens dans la zone d'étude varie en fonction des événements climatiques et des phénomènes de fracturations, qui permettent le transport 
rapide de l'eau à travers le substratum rocheux (rôle des linéaments).

Le péril fécal affecte les eaux par les excréments, soit directement en contact avec la nappe phréatique par l'intermédiaire des latrines inadéquates, soit transitant indirectement par écoulement souterrain latéral ou par infiltration verticale.

La composition bactériologique montre une forte concentration d'agents polluants, souvent innombrables tels que les coliformes totaux, Escherichia coli et streptocoques fécaux (en saison pluvieuse) dans les eaux des sources artésiennes. Il est à remarquer que la concentration d'agents polluants est nettement faible au niveau des forages artésiens. Néanmoins, la charge polluante excède les normes de l'OMS. L'absence des germes bactériens en saison sèche et leur présence subitement en saison pluvieuse de la même année illustre le rôle des linéaments. La présente étude avait pour but de tester une méthodologie pour l'interprétation de linéaments dans la relation entre eau de ruissellement et eau souterraine. La carte des linéaments obtenue pour la région de Zagnanado et de Zogbodomey nous permet de croire que celle-ci serait une zone de recharge pour les aquifères continus. La forte intersection et la forte densité retrouvée dans la commune de Zagnanado indiquent donc peut-être que l'écoulement pourrait être plus important dans cette zone. Cette situation ajoutée à la situation géographique des grandes dépressions qui abritent les sources malgré leur caractère artésien, font que les sources sont polluées toute l'année. Ce type d'étude est simple et efficace sans nécessairement être dispendieuse. Elle n'apporte pas nécessairement toutes les réponses, mais elle nous permet de tracer un bon portrait des fractures qui sont responsables de l'écoulement souterrain. Pour préciser le rôle et les particularités de la région de Zagnanado et de Zogbodomey, il fallait une étude de terrain, tel un relevé de fractures (carte géologique). Cela vient valider les résultats obtenus par cette étude d'interprétation de linéaments.

\section{REFERENCES}

Anderson FJ. 2009. Lineament mapping and analysis in the northeastern Williston Basin of North Dakota, pp. 24-25.

Dewandel B, Perrin J, Ahmed S, Aulong S, Hrkal Z, Lachassagne P, Samad M, Massuel S. 2010. Development of a tool for managing groundwater resources in semi-arid hard rock regions: application to a rural watershed in South India. Hydrological Processes, 24(19): 2784-2797.

Dewandel B, Maréchal JC, Bour O, Ladouche B, Ahmed S, Chandra S, Pauwels $\mathrm{H}$. 2012. Upscaling and regionalizing hydraulic conductivity and effective porosity at watershed scale in deeply weathered crystalline aquifers.

Elewa HH, Qaddah AA. 2011. Groundwater potentiality mapping in the Sinai Peninsula, Egypt, using remote sensing and GIS-watershed-based modeling. Hydrogeol. J., 19(3): 613-628.

Fernandes AJ, Rudolph DL. 2001. The influence of Cenozoic tectonics on the groundwater-production capacity of fractured zones: a case study in Sao Paulo, Brazil. Hydrogeol. J., 9: 151-167.

Gronayes CC, Koffi BP, Kouamé FK, Brou BK. 2003. Identification de couloirs de cisaillement à partir de l'imagerie tm de landsat: Application à l'Archéen de Blolequi (ouest de la Côte d'Ivoire). Télédétection, 3(2-3-4):217-226.

Hung LQ, Batelaan O, De Smedt F. 2005. Lineament extraction and analysis, comparision of LANDSAT ETM and ASTER imagerry. Case Study ; suoimuoi tropical Karst catchment, Vietnam. Proc. of SPIE, 5983: 1-12.

Kouamé KF, Lasm T, De Dreuzy JR, Akaffou AG, Bour O, Davy P. 2010. Contribution d'un modèle hydrogéologique stochastique à fractures discrètes à l'étude des aquifères fracturés 
du socle Archéen de Touba (Nord-Ouest de la Côte d'Ivoire). Revue des Sciences de l'Eau, 23(1): 41-56.

Kuusela-Lahtinen A, Niemi A, Luukkonen A, 2003. Flow Dimension as an Indicator of Hydraulic Behavior in Site Characterization of Fractured Rock. Groundwater, 41(3): 333-341.

Lachassagne P, Wyns R, Dewandel B, 2011. The fracture permeability of hard rock aquifers is due neither to tectonics, nor to uploading, but to weathering processes. Terra Nova, 23: 145-161.

Le Borgne T, Bour O, Paillet J-L, Caudal, JP. 2006. Assessment of preferential flow path connectivity, and hydraulic properties at single-borehole and crossborehole scales in a fractured aquifer. $J$. Hydrol., 328(1-2): 347-359.

Lee S, Kim YS, Oh HJ. 2012. Application of a weights-of-evidence method and GIS to regional groundwater productivity potential mapping. Journal of Environmental Management, 96(1): 91105.

Masoud A, Koike K. 2005. Remote sensing and GIS integration for groundwater potential mapping in Sinai Peninsula, Egypt. In: Cheng, Q., BonhamCarter, G. (Eds.), Annual Conference of the International-Association-for-

Mathematical-Geology. GIS and Spatial Analysis, York University, Toronto, 1-2: 440-445.

Naik PK, Awasthi AK, Anand AVSS, Mohan PC. 2001. Hydrogeologic framework of the Deccan terrain of the Koyna River basin, India. Hydrogeol. J., 9(3): 243264.

Negrel P, Pauwels H, Dewandel B, Gandolfi JM, Mascre C, Ahmed S. 2011. Understanding groundwater systems and their functioning through the study of stable water isotopes in a hard-rock aquifer (Maheshwaram watershed, India). J. Hydrol., 397(1-2): 55-70.
Neves MA, Morales N. 2007. Structural control over well productivity in the Jundiaí River catchment, Southeastern Brazil. Anais da Academia Brasileira de Ciências, 79(2): 307-320.

Oh HJ, Kim YS, Choi Y-S, Park E, Lee S. 2011. GIS mapping of regional probabilistic groundwater potential in the area of Pohang City, Korea. J. Hydrol., 399(3-4): 158-172.

Saley MB, Kouamé FK, Penven MJ, Biémi J, Boyossoro KH. 2005. Cartographie des zones à risque d'inondation dans la région semi- montagneuse à l'ouest de la Côte d'Ivoire: apports des MNA et de l'imagerie satellitaire. Télédétection, 5(12-3):53-67.

Sander P. 2007. Lineaments in groundwater exploration : a review of applications and limitations. Hydrogeol. J., 15: 71-74. Solomon S, Ghebreab W. 2008. Hardrock hydrotectonics using gis in the central highlands of Eritrea. Journal of Hydrology, 349: 147-155.

Taylor R, Howard K. 2000. A tectonogeomorphic model of the hydrogeology of deeply weathered crystalline rocks : Evidence from Uganda. Hydrogeol. J., 8: 279-294.

Walker DD, Gylling B, Ström A, Selroos, JO. 2001. Hydrogeologic studies for nuclearwaste disposal in Sweden. Hydrogeol. J., 9: 419-431.

watershed scale in deeply weathered crystalline aquifers. J. Hydrol., 416-417: 83-97.

Givord L, Dorioz JM. 2010 La survie des microorganismes d'origine fécale dans les effluents et les sols. INRA thonon Umr carrtel. Etude réalisée dans le cadre du projet CasDAR Territ'Eau, 23 p.

Majdoub R, Cote C, Labidi M, Guay K, généreux M. 2003. Impact de l'utilisation des engrais de ferme sur la qualité microbiologique de l'eau souterraine (Revue de littérature). IRDA (Institut de recherche et de développement en agroenvironnement): Canada. $125 \mathrm{p}$.

J-B. Achidi L, Bourguet R, Elsaesser A, Legier E, Paulvé N, tribouillard. 2012. 
Carte Hydrogeologique Du Benin. Programme Eau Potable et Assainissement (PEP). DG eau direction generale de l'eau Ministère de l'énergie, des recherches pétrolières et minières, de l'eau et du développement des énergies renouvelables (merpmeder), $77 \mathrm{p}$.

Le Barbé L, Alé G, Millet B, Texier H, Borel Y, Gualde R. 1993. Les Ressources en Eaux Superficielles de la République du Bénin. Edition Orstom : Paris ; 540 p.

Scanvic JY. 1993. Utilisation de la télédétection dans les sciences de la terre. Manuels et méthodes, BRGM, Orléans, $284 \mathrm{p}$.

Singhal BBS, Gupta RP. 2010. Applied Hydrogeology of Fractured Rocks ( $2^{\text {nd }}$ edn). Kluwer Academic Publishers (Springer) : Dordrecht, Netherlands ; 428.

Babaye MSA. 2012. Evaluation des ressources en eau souterraines dans le bassin de Dargol (Liptako- Niger). Thèses Doct. ès Sci., Univ. De Liège. Univ Abdou Moumouni. Faculté de Sciences et Techniques. Département de Géologie Niger, $244 \mathrm{p}$.

Durand. 2005 : Recherche multidisciplinaire pour caractériser deux aquifères fracturés : les eaux minérales de Plancoët en contexte métamorphique, et de Quezac en milieu carbonaté : Thèse Univ. Université Paris- Pierre et Marie Curie, $255 \mathrm{p}$.

Jourda JPP. 2005. Méthodologie d'application des techniques de télédétection et des systèmes d'information géographique à l'étude des aquifères fissurés d'Afrique de l'ouest. Concept de l'hydrotechnique spatiale: cas des zones tests de la Côte d'Ivoire. Thèse de Doctorat d'état, Univ, Cocody, 403 p.

Razack M. 1984. Application de méthodes numériques et statistiques à l'identification des réservoirs fissurés carbonatés en hydrogéologie. Thèses Doct. ès Sci., Univ. Languedoc, 384 p.

Singhal BBS, Gupta RP. 2010. Applied Hydrogeology of Fractured Rocks, Kluwer Academic Publishers: Dordrecht, $\mathrm{NL}$.

Sterckx A. 2013. Étude des facteurs influençant le rendement des puits d'alimentation de particuliers qui exploitent le roc fracturé en Outaouais. Maîtrise interuniversitaire en Sciences de la Terre Maître ès Sciences (M.Sc.). Université Laval. Québec, Canada, 74 p.

Totin HS. 2010. Sensibilité des eaux souterraines du bassin sédimentaire côtier du Bénin à l'évolution du climat et aux modes d'exploitation : stratégies de gestion durable. Thèse de Doctorat de l'Université d'Abomey-Calavi. Bénin, $283 \mathrm{p}$.

Yaméogo OS. 2008. Ressources en eau souterraine $\mathrm{du}$ centre urbain de Ouagadougou au Burkina Faso, qualité et vulnérabilité ; Univ. Avignon et pays de Vaucluse, Univ. Ouagadougou, 245 p. 